\title{
An algorithm for a common fixed point of a family of pseudocontractive mappings
}

Habtu Zegeye ${ }^{1}$ and Naseer Shahzad ${ }^{2 *}$

\section{"Correspondence:}

nshahzad@kau.edu.sa

${ }^{2}$ Department of Mathematics, King

Abdulaziz University, P.O. Box 80203,

Jeddah, 21589, Saudi Arabia

Full list of author information is

available at the end of the article

\begin{abstract}
We introduce an iterative process which converges strongly to a common fixed point of a family of Lipschitzian pseudocontractive mappings. Our theorems improve and unify most of the results that have been proved for this important class of nonlinear operators.

MSC: 47H09; 47J20;65J15

Keywords: fixed points of mappings; monotone mappings; pseudocontractive mappings; strong convergence
\end{abstract}

\section{Introduction}

Let $C$ be a nonempty subset of a real Hilbert space $H$. A mapping $T: C \rightarrow H$ is called pseudocontractive if and only if

$$
\|T x-T y\|^{2} \leq\|x-y\|^{2}+\|(I-T) x-(I-T) y\|^{2}, \quad \text { for all } x, y \in C,
$$

and $T$ is called $\alpha$-strictly pseudocontractive [1] if and only if there exists $\alpha \in[0,1)$ such that

$$
\|T x-T y\|^{2} \leq\|x-y\|^{2}+\alpha\|(I-T) x-(I-T) y\|^{2}, \quad \text { for all } x, y \in C,
$$

$T$ is called Lipschitzian if and only if

$$
\|T x-T y\| \leq L\|x-y\|, \quad \text { for all } x, y \in K .
$$

If in (1.3) we have $L \leq 1$, then $T$ is called nonexpansive. We note that inequalities (1.1) and (1.2) can be equivalently written as

$$
\langle x-y, T x-T y\rangle \leq\|x-y\|^{2}, \quad \text { for all } x, y \in C
$$

and

$$
\langle x-y, T x-T y\rangle \leq\|x-y\|^{2}-\lambda\|(I-T) x-(I-T) y\|^{2},
$$

for some $\lambda>0$, respectively.

(0) 2013 Zegeye and Shahzad; licensee Springer. This is an Open Access article distributed under the terms of the Creative Commons Attribution License (http://creativecommons.org/licenses/by/2.0), which permits unrestricted use, distribution, and reproduction in any medium, provided the original work is properly cited. 
We observe from (1.1), (1.2) and (1.3) that every nonexpansive mapping is an $\alpha$-strictly pseudocontractive mapping and every $\alpha$-strictly pseudocontractive mapping is a pseudocontractive mapping, and hence a class of pseudocontractive mappings is a more general class of mappings.

Furthermore, pseudocontractive mappings are related with the important class of nonlinear monotone mappings, where a mapping $A$ with domain $D(A)$ and range $R(A)$ in $H$ is called monotone if the inequality

$$
\langle x-y, A x-A y\rangle \geq 0,
$$

holds for every $x, y \in D(A)$. We note that $T$ is pseudocontractive if and only if $A:=I-T$ is monotone, and hence a fixed point of $T, F(T):=\{x \in D(T): T x=x\}$, is a zero of $A$, $N(A):=\{x \in D(A): A x=0\}$. It is now well known (see, e.g., [2]) that if $A$ is monotone, then the solutions of the equation $A x=0$ correspond to the equilibrium points of some evolution systems.

Finding a point in the intersection of fixed point sets of a family of nonexpansive mappings is a task that occurs frequently in various areas of mathematical sciences and engineering. For example, the well-known convex feasibility problem reduces to finding a point in the intersection of fixed point sets of a family of nonexpansive mappings; see, e.g., $[3,4]$.

Consequently, considerable research efforts have been devoted to developing iterative methods for approximating a common fixed point (when it exists) for a family of nonexpansive mappings and $\alpha$-strictly pseudocontractive mappings. Bauschke [5] was the first to introduce a Halpern-type iterative process (see, e.g., [6]) for approximating a common fixed point for a finite family of $N$ nonexpansive self-mappings. He proved the following theorem.

Theorem BSK (Bauschke [5], Theorem 3.1) Let $K$ be a nonempty closed convex subset of a Hilbert space $H$, and let $T_{1}, T_{2}, \ldots, T_{N}$ be a finite family of nonexpansive mappings of $K$ into itself with $F:=\bigcap_{i=1}^{N} F\left(T_{i}\right) \neq \emptyset$ and $F=F\left(T_{N} T_{N-1} \cdots T_{1}\right)=F\left(T_{1} T_{N} \cdots T_{2}\right)=$ $F\left(T_{N-1} T_{N-2} \cdots T_{1} T_{N}\right)$. Given points $u, x_{0} \in C$, let $\left\{x_{n}\right\}$ be generated by

$$
x_{n+1}:=\alpha_{n+1} u+\left(1-\alpha_{n+1}\right) T_{n+1} x_{n}, \quad n \geq 0,
$$

where $T_{n}:=T_{n(\bmod N)}$ and $\left\{\alpha_{n}\right\} \subset(0,1)$ satisfies $\sum_{n \geq 1}\left|\alpha_{n+r}-\alpha_{n}\right|<\infty$. Then $\left\{x_{n}\right\}$ converges strongly to $P_{F} u$, where $P_{F}: H \rightarrow F$ is the metric projection.

Various authors have studied iterative schemes similar to that of Theorem BSK in more general Banach spaces on the one hand, and using various conditions on the sequence $\left\{\alpha_{n}\right\}$ on the other hand (see, for example, Colao et al. [7], Yao [8], Takahashi and Takahashi [9], Plubtieng and Punpaeng [10], Ceng et al. [11] and the references therein). Many authors have also studied iterative methods for a family of $\alpha$-strictly pseudocontractive mappings (see, e.g., [12-15] and the references therein).

Our concern now is the following: Can we construct an iterative sequence for a common fixed point of a finite family of pseudocontractive mappings?

In 2008, Zhou [16] studied weak convergence of an implicit scheme to a common fixed point of a finite family of pseudocontractive mappings in Banach spaces more general than 
Hilbert spaces. Let $C$ be a closed convex subset of $E$, and let $\left\{T_{i}\right\}_{i=1}^{N}$ be a finite family of Lipschitzian pseudocontractive self-mappings of $C$ subset of $E$ which is a real uniformly convex Banach space with a Frêchet differentiable norm and $F:=\bigcap_{i=1}^{N} F\left(T_{i}\right) \neq \emptyset$. Let $\left\{x_{n}\right\}$ be defined by

$$
x_{n}=\alpha_{n} x_{n-1}+\left(1-\alpha_{n}\right) T_{n} x_{n}, \quad n \geq 1,
$$

where $T_{n}=T_{n(\bmod N)}$. He proved that $\left\{x_{n}\right\}$ converges weakly to a common fixed point of the family $\left\{T_{i}\right\}_{i=1}^{N}$ under certain conditions on the parameter $\left\{\alpha_{n}\right\}$.

We remark that the scheme in the theorem of Zhou [16] is implicit and the convergence is weak convergence.

Recently, Zegeye et al. [17] proved the following strong convergence of Ishikawa iterative process [18] for a common fixed point of a finite family of Lipschitz pseudocontractive mappings.

Theorem ZSA [17] Let $C$ be a nonempty, closed and convex subset of a real Hilbert space $H$. Let $T_{i}: C \rightarrow C, i=1,2, \ldots, N$, be a finite family of Lipschitz pseudocontractive mappings with Lipschitzian constants $L_{i}$, for $i=1,2, \ldots, N$, respectively. Assume that the interior of $F:=\bigcap_{i=1}^{N} F\left(T_{i}\right)$ is nonempty. Let $\left\{x_{n}\right\}$ be a sequence generated from an arbitrary $x_{0} \in E$ by

$$
\left\{\begin{array}{l}
y_{n}=\left(1-\beta_{n}\right) x_{n}+\beta_{n} T_{n} x_{n}, \\
x_{n+1}=\left(1-\alpha_{n}\right) x_{n}+\alpha_{n} T_{n} y_{n}, \quad n \geq 1,
\end{array}\right.
$$

where $T_{n}:=T_{n(\bmod N)}$ and $\left\{\alpha_{n}\right\},\left\{\beta_{n}\right\} \subset(0,1)$ satisfy certain appropriate conditions. Then $\left\{x_{n}\right\}$ converges strongly to a common fixed point of $\left\{T_{1}, T_{2}, \ldots, T_{N}\right\}$.

It is worth to mention that the assumption 'interior of $F(T)$ is nonempty' in Theorem ZSA is severe restriction.

More recently, Daman and Zegeye [19] proved the following strong convergence of a Halpern-type [6] iterative process for a common fixed point of a finite family of Lipschitz pseudocontractive mappings under the assumption that the family satisfies condition $(H)$, that is, the family $\left\{T_{i}: i=1,2, \ldots, N\right\}$ of pseudocontractive mappings is said to satisfy condition $(H)$ if and only if $\left\langle T_{i} x-x, T_{j} x-x\right\rangle \geq 0$ for $i, j \in\{1,2, \ldots, N\}$. We can also see that condition $(H)$ is again severe restriction.

It is our purpose in this paper to introduce an iterative scheme which converges strongly to a common fixed point of a family of Lipschitz pseudocontractive mappings. The assumption that interior of $F(T)$ is nonempty or condition $(H)$ is dispensed with. The results obtained in this paper improve and extend the results of Zhou [20], Theorem ZSA, Daman and Zegeye [19] and some other results in this direction.

\section{Preliminaries}

In what follows we shall make use of the following lemmas.

Lemma 2.1 Let $H$ be a real Hilbert space. Then, for any given $x, y \in E$, the following inequality holds:

$$
\|x+y\|^{2} \leq\|x\|^{2}+2\langle y, x+y\rangle .
$$


Lemma 2.2 [21] Let $C$ be a convex subset of a real Hilbert space H. Let $x \in H$. Then $x_{0}=$ $P_{C} x$ if and only if

$$
\left\langle z-x_{0}, x-x_{0}\right\rangle \leq 0, \quad \forall z \in C
$$

Lemma 2.3 [22] Let $\left\{a_{n}\right\}$ be a sequence of nonnegative real numbers satisfying the following relation:

$$
a_{n+1} \leq\left(1-\alpha_{n}\right) a_{n}+\alpha_{n} \delta_{n}, \quad n \geq n_{0},
$$

where $\left\{\alpha_{n}\right\} \subset(0,1)$ and $\left\{\delta_{n}\right\} \subset R$ satisfy the following conditions: $\lim _{n \rightarrow \infty} \alpha_{n}=0, \sum_{n=1}^{\infty} \alpha_{n}=$ $\infty$, and $\lim \sup _{n \rightarrow \infty} \delta_{n} \leq 0$. Then $\lim _{n \rightarrow \infty} a_{n}=0$.

Lemma 2.4 [23] Let $H$ be a real Hilbert space, $C$ be a closed convex subset of $H$ and $T$ : $C \rightarrow C$ be a continuous pseudocontractive mapping. Then

(i) $F(T)$ is a closed convex subset of $C$;

(ii) $(I-T)$ is demiclosed at zero, i.e., if $\left\{x_{n}\right\}$ is a sequence in $C$ such that $x_{n} \rightarrow x$ and $T x_{n}-x_{n} \rightarrow 0$, as $n \rightarrow \infty$, then $x=T(x)$.

Lemma 2.5 [24] Let $\left\{a_{n}\right\}$ be sequences of real numbers such that there exists a subsequence $\left\{n_{i}\right\}$ of $\{n\}$ such that $a_{n_{i}}<a_{n_{i}+1}$ for all $i \in N$. Then there exists a nondecreasing sequence $\left\{m_{k}\right\} \subset N$ such that $m_{k} \rightarrow \infty$ and the following properties are satisfied by all (sufficiently large) numbers $k \in N$ :

$$
a_{m_{k}} \leq a_{m_{k}+1} \text { and } a_{k} \leq a_{m_{k}+1}
$$

In fact, $m_{k}$ is the largest number $n$ in the set $\{1,2, \ldots, k\}$ such that the condition $a_{n} \leq a_{n+1}$ holds.

Lemma 2.6 [25] Let $H$ be a real Hilbert space. Then, for all $x_{i} \in H$ and $\alpha_{i} \in[0,1]$ for $i=1,2, \ldots, n$ such that $\alpha_{1}+\alpha_{2}+\cdots+\alpha_{n}=1$, the following equality holds:

$$
\left\|\alpha_{0} x_{0}+\alpha_{1} x_{1}+\cdots+\alpha_{n} x_{n}\right\|^{2}=\sum_{i=0}^{n} \alpha_{i}\left\|x_{i}\right\|^{2}-\sum_{0 \leq i, j \leq n} \alpha_{i} \alpha_{j}\left\|x_{i}-x_{j}\right\|^{2} .
$$

\section{Main result}

Theorem 3.1 Let $C$ be a nonempty, closed and convex subset of a real Hilbert space $H$. Let $T_{1}, T_{2}: C \rightarrow C$ be Lipschitz pseudocontractive mappings with Lipschitz constants $L_{1}$ and $L_{2}$, respectively. Assume that $\mathcal{F}=F\left(T_{1}\right) \cap F\left(T_{2}\right)$ is nonempty. Let a sequence $\left\{x_{n}\right\}$ be generated from an arbitrary $x_{0}, u \in C$ by

$$
\left\{\begin{array}{l}
z_{n}=\left(1-c_{n}\right) x_{n}+c_{n} T_{2} x_{n} ; \\
y_{n}=\left(1-\beta_{n}\right) x_{n}+\beta_{n} T_{1} x_{n} ; \\
x_{n+1}=\alpha_{n} u+\left(1-\alpha_{n}\right)\left(\theta_{n} x_{n}+\delta_{n} T_{1} y_{n}+\gamma_{n} T_{2} z_{n}\right), \quad n \geq 0,
\end{array}\right.
$$

where $\left\{\delta_{n}\right\},\left\{\theta_{n}\right\},\left\{\gamma_{n}\right\} \subset(a, b) \subset(0,1),\left\{\alpha_{n}\right\} \subset(0, c) \subset(0,1)$ satisfy the following conditions: (i) $\theta_{n}+\delta_{n}+\gamma_{n}=1$; (ii) $\lim _{n \rightarrow \infty} \alpha_{n}=0, \sum \alpha_{n}=\infty$; (iii) $\delta_{n}+\gamma_{n} \leq c_{n}, \beta_{n} \leq \beta<\frac{1}{\sqrt{1+L^{2}}+1}, \forall n \geq 1$, 
for $L:=\max \left\{L_{1}, L_{2}\right\}$. Then $\left\{x_{n}\right\}$ converges strongly to a common fixed point of $T_{1}$ and $T_{2}$ nearest to $u$.

Proof We make use of some ideas of the paper [19]. Let $p \in \mathcal{F}$. Then, from (3.1), (1.1) and Lemma 2.6, we have that

$$
\begin{aligned}
\left\|x_{n+1}-p\right\|^{2}= & \left\|\alpha_{n} u+\left(1-\alpha_{n}\right)\left(\theta_{n} x_{n}+\delta_{n} T_{1} y_{n}+\gamma_{n} T_{2} z_{n}\right)-p\right\|^{2} \\
\leq & \alpha_{n}\|u-p\|^{2}+\left(1-\alpha_{n}\right)\left\|\delta_{n}\left(T_{1} y_{n}-p\right)+\theta_{n}\left(x_{n}-p\right)+\gamma_{n}\left(T_{2} z_{n}-p\right)\right\|^{2} \\
\leq & \alpha_{n}\|u-p\|^{2}+\left(1-\alpha_{n}\right)\left[\delta_{n}\left\|T_{1} y_{n}-p\right\|^{2}+\theta_{n}\left\|x_{n}-p\right\|^{2}\right. \\
& \left.+\gamma_{n}\left\|T_{2} z_{n}-p\right\|^{2}\right]-\left(1-\alpha_{n}\right) \delta_{n} \theta_{n}\left\|T_{1} y_{n}-x_{n}\right\|^{2} \\
& -\left(1-\alpha_{n}\right) \theta_{n} \gamma_{n}\left\|T_{2} z_{n}-x_{n}\right\|^{2},
\end{aligned}
$$

and hence

$$
\begin{aligned}
\left\|x_{n+1}-p\right\|^{2} \leq & \alpha_{n}\|u-p\|^{2}+\left(1-\alpha_{n}\right) \delta_{n}\left[\left\|y_{n}-p\right\|^{2}+\left\|y_{n}-T_{1} y_{n}\right\|^{2}\right] \\
& +\left(1-\alpha_{n}\right) \theta_{n}\left\|x_{n}-p\right\|^{2}+\left(1-\alpha_{n}\right) \gamma_{n}\left[\left\|z_{n}-p\right\|^{2}+\left\|z_{n}-T_{2} z_{n}\right\|^{2}\right] \\
& -\left(1-\alpha_{n}\right) \delta_{n} \theta_{n}\left\|T_{1} y_{n}-x_{n}\right\|^{2}-\left(1-\alpha_{n}\right) \theta_{n} \gamma_{n}\left\|T_{2} z_{n}-x_{n}\right\|^{2} \\
\leq & \alpha_{n}\|u-p\|^{2}+\left(1-\alpha_{n}\right) \delta_{n}\left\|y_{n}-p\right\|^{2}+\left(1-\alpha_{n}\right) \delta_{n}\left\|y_{n}-T_{1} y_{n}\right\|^{2} \\
& +\left(1-\alpha_{n}\right) \theta_{n}\left\|x_{n}-p\right\|^{2}+\left(1-\alpha_{n}\right) \gamma_{n}\left\|z_{n}-p\right\|^{2} \\
& +\left(1-\alpha_{n}\right) \gamma_{n}\left\|z_{n}-T_{2} z_{n}\right\|^{2}-\left(1-\alpha_{n}\right) \delta_{n} \theta_{n}\left\|T_{1} y_{n}-x_{n}\right\|^{2} \\
& -\left(1-\alpha_{n}\right) \theta_{n} \gamma_{n}\left\|T_{2} z_{n}-x_{n}\right\|^{2} .
\end{aligned}
$$

In addition, from (3.1), Lemma 2.6 and (1.1), we get that

$$
\begin{aligned}
\left\|y_{n}-p\right\|^{2}= & \left\|\left(1-\beta_{n}\right)\left(x_{n}-p\right)+\beta_{n}\left(T_{1} x_{n}-p\right)\right\|^{2} \\
= & \left(1-\beta_{n}\right)\left\|x_{n}-p\right\|^{2}+\beta_{n}\left\|T_{1} x_{n}-p\right\|^{2} \\
& -\beta_{n}\left(1-\beta_{n}\right)\left\|x_{n}-T_{1} x_{n}\right\|^{2} \\
\leq & \left(1-\beta_{n}\right)\left\|x_{n}-p\right\|^{2}+\beta_{n}\left[\left\|x_{n}-p\right\|^{2}+\left\|x_{n}-T_{1} x_{n}\right\|^{2}\right] \\
& -\beta_{n}\left(1-\beta_{n}\right)\left\|x_{n}-T_{1} x_{n}\right\|^{2} \\
= & \left\|x_{n}-p\right\|^{2}+\beta_{n}^{2}\left\|x_{n}-T_{1} x_{n}\right\|^{2} .
\end{aligned}
$$

Similarly, we have that

$$
\left\|z_{n}-p\right\|^{2}=\left\|x_{n}-p\right\|^{2}+c_{n}^{2}\left\|x_{n}-T_{2} x_{n}\right\|^{2} .
$$

Furthermore, from (3.1) and Lemma 2.6, we have that

$$
\begin{aligned}
\left\|y_{n}-T_{1} y_{n}\right\|^{2} & =\left\|\left(1-\beta_{n}\right)\left(x_{n}-T_{1} y_{n}\right)+\beta_{n}\left(T_{1} x_{n}-T_{1} y_{n}\right)\right\|^{2} \\
& =\left(1-\beta_{n}\right)\left\|x_{n}-T_{1} y_{n}\right\|^{2}+\beta_{n}\left\|T_{1} x_{n}-T_{1} y_{n}\right\|^{2}
\end{aligned}
$$




$$
\begin{aligned}
& -\beta_{n}\left(1-\beta_{n}\right)\left\|x_{n}-T_{1} x_{n}\right\|^{2} \\
\leq & \left(1-\beta_{n}\right)\left\|x_{n}-T_{1} y_{n}\right\|^{2}+\beta_{n} L^{2}\left\|x_{n}-y_{n}\right\|^{2} \\
& -\beta_{n}\left(1-\beta_{n}\right)\left\|x_{n}-T_{1} x_{n}\right\|^{2} \\
= & \left(1-\beta_{n}\right)\left\|x_{n}-T_{1} y_{n}\right\|^{2}+\beta_{n}^{3} L^{2}\left\|x_{n}-T_{1} x_{n}\right\|^{2} \\
& -\beta_{n}\left(1-\beta_{n}\right)\left\|x_{n}-T_{1} x_{n}\right\|^{2} \\
= & \left(1-\beta_{n}\right)\left\|x_{n}-T_{1} y_{n}\right\|^{2}-\beta_{n}\left(1-L^{2} \beta_{n}^{2}-\beta_{n}\right)\left\|x_{n}-T_{1} x_{n}\right\|^{2} .
\end{aligned}
$$

Similarly, we obtain that

$$
\left\|z_{n}-T_{2} z_{n}\right\|^{2}=\left(1-c_{n}\right)\left\|x_{n}-T_{2} z_{n}\right\|^{2}-c_{n}\left(1-L^{2} c_{n}^{2}-c_{n}\right)\left\|x_{n}-T_{2} x_{n}\right\|^{2} .
$$

Substituting (3.3), (3.4), (3.5) and (3.6) into (3.2), we obtain that

$$
\begin{aligned}
\left\|x_{n+1}-p\right\|^{2} \leq & \alpha_{n}\|u-p\|^{2}+\left(1-\alpha_{n}\right) \delta_{n}\left[\left\|x_{n}-p\right\|^{2}+\beta_{n}^{2}\left\|x_{n}-T_{1} x_{n}\right\|^{2}\right] \\
& +\left(1-\alpha_{n}\right) \delta_{n}\left[\left(1-\beta_{n}\right)\left\|x_{n}-T_{1} y_{n}\right\|^{2}-\beta_{n}\left(1-L^{2} \beta_{n}^{2}-\beta_{n}\right)\right. \\
& \left.\times\left\|x_{n}-T_{1} x_{n}\right\|^{2}\right]+\left(1-\alpha_{n}\right) \theta_{n}\left\|x_{n}-p\right\|^{2}+\left(1-\alpha_{n}\right) \gamma_{n}\left[\left\|x_{n}-p\right\|^{2}\right. \\
& \left.+c_{n}^{2}\left\|x_{n}-T_{2} x_{n}\right\|^{2}\right]+\left(1-\alpha_{n}\right) \gamma_{n}\left[\left(1-c_{n}\right)\left\|x_{n}-T_{2} z_{n}\right\|^{2}\right. \\
& \left.-c_{n}\left(1-L^{2} c_{n}^{2}-c_{n}\right)\left\|x_{n}-T_{2} x_{n}\right\|^{2}\right]-\left(1-\alpha_{n}\right) \delta_{n} \theta_{n}\left\|T_{1} y_{n}-x_{n}\right\|^{2} \\
& -\left(1-\alpha_{n}\right) \theta_{n} \gamma_{n}\left\|T_{2} z_{n}-x_{n}\right\|^{2} \\
= & \alpha_{n}\|u-p\|^{2}+\left(1-\alpha_{n}\right)\left\|x_{n}-p\right\|^{2}-\left(1-\alpha_{n}\right) \delta_{n} \beta_{n} \\
& \times\left(1-\left(L^{2} \beta_{n}^{2}+2 \beta_{n}\right)\right)\left\|x_{n}-T_{1} x_{n}\right\|^{2} \\
& -\left(1-\alpha_{n}\right) \gamma_{n} c_{n}\left(1-\left(L^{2} c_{n}^{2}+2 c_{n}\right)\right)\left\|x_{n}-T_{2} x_{n}\right\|^{2} \\
& +\left(1-\alpha_{n}\right) \delta_{n}\left(1-\theta_{n}-\beta_{n}\right)\left\|T_{1} y_{n}-x_{n}\right\|^{2} \\
& +\left(1-\alpha_{n}\right) \gamma_{n}\left(1-\theta_{n}-c_{n}\right)\left\|T_{2} z_{n}-x_{n}\right\|^{2},
\end{aligned}
$$

and hence

$$
\begin{aligned}
\left\|x_{n+1}-p\right\|^{2} \leq & \alpha_{n}\|u-p\|^{2}+\left(1-\alpha_{n}\right)\left\|x_{n}-p\right\|^{2}-\left(1-\alpha_{n}\right) \delta_{n} \beta_{n} \\
& \times\left(1-\left(L^{2} \beta_{n}^{2}+2 \beta_{n}\right)\right)\left\|x_{n}-T_{1} x_{n}\right\|^{2} \\
& -\left(1-\alpha_{n}\right) \gamma_{n} c_{n}\left(1-\left(L^{2} c_{n}^{2}+2 c_{n}\right)\right)\left\|x_{n}-T_{2} x_{n}\right\|^{2} \\
& +\left(1-\alpha_{n}\right) \delta_{n}\left(\delta_{n}+\gamma_{n}-\beta_{n}\right)\left\|T_{1} y_{n}-x_{n}\right\|^{2} \\
& +\left(1-\alpha_{n}\right) \gamma_{n}\left(\delta_{n}+\gamma_{n}-c_{n}\right)\left\|T_{2} z_{n}-x_{n}\right\|^{2} .
\end{aligned}
$$

Now, from (iii) of the hypothesis, we have that

$$
\begin{aligned}
& 1-2 \beta_{n}-L^{2} \beta_{n}^{2} \geq 1-2 \beta-L^{2} \beta^{2}>0, \\
& 1-2 c_{n}-L^{2} c_{n}^{2} \geq 1-2 \beta-L^{2} \beta^{2}>0
\end{aligned}
$$


and

$$
\left(\delta_{n}+\gamma_{n}\right)-\beta_{n} \leq 0, \quad\left(\delta_{n}+\gamma_{n}\right)-c_{n} \leq 0 \quad \text { for all } n \geq 1 .
$$

Thus, inequality (3.7) implies that

$$
\left\|x_{n+1}-p\right\|^{2} \leq \alpha_{n}\|u-p\|^{2}+\left(1-\alpha_{n}\right)\left\|x_{n}-p\right\|^{2} .
$$

Thus, by induction,

$$
\left\|x_{n+1}-p\right\|^{2} \leq \max \left\{\|u-p\|^{2},\left\|x_{0}-p\right\|^{2}\right\}, \quad \forall n \geq 0
$$

which implies that $\left\{x_{n}\right\}$ and hence $\left\{y_{n}\right\}$ are bounded.

Let $x^{*}=P_{\mathcal{F}}(u)$. Then, using (3.1), Lemma 2.1 and following the methods used to get (3.7), we obtain that

$$
\begin{aligned}
\left\|x_{n+1}-x^{*}\right\|^{2}= & \left\|\alpha_{n} u+\left(1-\alpha_{n}\right)\left(\theta_{n} x_{n}+\delta_{n} T_{1} y_{n}+\gamma_{n} T_{2} z_{n}\right)-x^{*}\right\|^{2} \\
= & \left\|\alpha_{n}\left(u-x^{*}\right)+\left(1-\alpha_{n}\right)\left[\theta_{n} x_{n}+\delta_{n} T_{1} y_{n}+\gamma_{n} T_{2} z_{n}-x^{*}\right]\right\|^{2} \\
\leq & \left(1-\alpha_{n}\right)\left\|\delta_{n} T_{1} y_{n}+\theta_{n} x_{n}+\gamma_{n} T_{2} z_{n}-x^{*}\right\|^{2} \\
& +2 \alpha_{n}\left\langle u-x^{*}, x_{n+1}-x^{*}\right\rangle \\
\leq & \left(1-\alpha_{n}\right) \delta_{n}\left\|T_{1} y_{n}-x^{*}\right\|^{2}+\left(1-\alpha_{n}\right) \theta_{n}\left\|x_{n}-x^{*}\right\|^{2} \\
& +\left(1-\alpha_{n}\right) \gamma_{n}\left\|T_{2} z_{n}-x^{*}\right\|^{2}-\left(1-\alpha_{n}\right) \theta_{n} \delta_{n}\left\|T_{1} y_{n}-x_{n}\right\|^{2} \\
& -\left(1-\alpha_{n}\right) \theta_{n} \gamma_{n}\left\|T_{2} z_{n}-x_{n}\right\|^{2}+2 \alpha_{n}\left\langle u-x^{*}, x_{n+1}-x^{*}\right\rangle
\end{aligned}
$$

and

$$
\begin{aligned}
\left\|x_{n+1}-x^{*}\right\|^{2} \leq & \left(1-\alpha_{n}\right) \delta_{n}\left[\left\|y_{n}-x^{*}\right\|^{2}+\left\|y_{n}-T_{1} y_{n}\right\|^{2}\right] \\
& +\left(1-\alpha_{n}\right) \theta_{n}\left\|x_{n}-x^{*}\right\|^{2}+\left(1-\alpha_{n}\right) \gamma_{n}\left[\left\|z_{n}-x^{*}\right\|^{2}\right. \\
& \left.+\left\|z_{n}-T_{2} z_{n}\right\|^{2}\right]-\left(1-\alpha_{n}\right) \theta_{n} \delta_{n}\left\|T_{1} y_{n}-x_{n}\right\|^{2} \\
& -\left(1-\alpha_{n}\right) \theta_{n} \gamma_{n}\left\|T_{2} z_{n}-x_{n}\right\|^{2}+2 \alpha_{n}\left\langle u-x^{*}, x_{n+1}-x^{*}\right\rangle \\
\leq & \left(1-\alpha_{n}\right) \delta_{n}\left[\left\|x_{n}-x^{*}\right\|^{2}+\beta_{n}^{2}\left\|x_{n}-T_{1} x_{n}\right\|^{2}\right] \\
& +\left(1-\alpha_{n}\right) \delta_{n}\left[\left(1-\beta_{n}\right)\left\|x_{n}-T_{1} y_{n}\right\|^{2}-\beta_{n}\left(1-L^{2} \beta_{n}^{2}-\beta_{n}\right)\right. \\
& \left.\times\left\|x_{n}-T_{1} x_{n}\right\|^{2}\right]+\left(1-\alpha_{n}\right) \theta_{n}\left\|x_{n}-x^{*}\right\|^{2} \\
& +\left(1-\alpha_{n}\right) \gamma_{n}\left[\left\|x_{n}-x^{*}\right\|^{2}+c_{n}^{2}\left\|x_{n}-T_{2} x_{n}\right\|^{2}\right] \\
& +\left(1-\alpha_{n}\right) \gamma_{n}\left[\left(1-c_{n}\right)\left\|x_{n}-T_{2} z_{n}\right\|^{2}-c_{n}\left(1-L^{2} c_{n}^{2}-c_{n}\right)\right. \\
& \left.\times\left\|x_{n}-T_{2} x_{n}\right\|^{2}\right]-\left(1-\alpha_{n}\right) \theta_{n} \delta_{n}\left\|T_{1} y_{n}-x_{n}\right\|^{2}-\left(1-\alpha_{n}\right) \theta_{n} \gamma_{n} \\
& \times\left\|T_{2} z_{n}-x_{n}\right\|^{2}+2 \alpha_{n}\left\langle u-x^{*}, x_{n+1}-x^{*}\right\rangle,
\end{aligned}
$$


which implies that

$$
\begin{aligned}
\left\|x_{n+1}-x^{*}\right\|^{2} \leq & \left(1-\alpha_{n}\right)\left\|x_{n}-x^{*}\right\|^{2}-\left(1-\alpha_{n}\right) \delta_{n} \beta_{n}\left[1-L^{2} \beta_{n}^{2}-2 \beta_{n}\right] \\
& \times\left\|x_{n}-T_{1} x_{n}\right\|^{2}+\left(1-\alpha_{n}\right) \delta_{n}\left(\delta_{n}+\gamma_{n}-\beta_{n}\right)\left\|x_{n}-T_{1} y_{n}\right\|^{2} \\
& -\left(1-\alpha_{n}\right) \delta_{n} c_{n}\left[1-L^{2} c_{n}^{2}-2 c_{n}\right]\left\|x_{n}-T_{2} x_{n}\right\|^{2} \\
& +\left(1-\alpha_{n}\right) \gamma_{n}\left(\delta_{n}+\gamma_{n}-c_{n}\right)\left\|x_{n}-T_{2} z_{n}\right\|^{2}+2 \alpha_{n}\left\langle u-x^{*}, x_{n+1}-x^{*}\right\rangle \\
\leq & \left(1-\alpha_{n}\right)\left\|x_{n}-x^{*}\right\|^{2}+2 \alpha_{n}\left\langle u-x^{*}, x_{n+1}-x^{*}\right\rangle .
\end{aligned}
$$

Now, we consider two cases.

Case 1. Suppose that there exists $n_{0} \in \mathbb{N}$ such that $\left\{\left\|x_{n}-x^{*}\right\|\right\}$ is decreasing for all $n \geq n_{0}$. Then, we get that $\left\{\left\|x_{n}-x^{*}\right\|\right\}$ is convergent. Thus, from (3.12), (3.8), (3.9) and (3.10), we have that

$$
x_{n}-T_{1} x_{n} \rightarrow 0, \quad x_{n}-T_{2} x_{n} \rightarrow 0 \quad \text { as } n \rightarrow \infty .
$$

Furthermore, since $\left\{x_{n+1}\right\}$ is a bounded subset of $H$ which is reflexive, we can choose a subsequence $\left\{x_{n_{i}+1}\right\}$ of $\left\{x_{n+1}\right\}$ such that $x_{n_{i}+1} \rightarrow x$ and $\limsup _{n \rightarrow \infty}\left\langle u-x^{*}, x_{n+1}-x^{*}\right\rangle=$ $\lim _{i \rightarrow \infty}\left\langle u-x^{*}, x_{n_{i}+1}-x^{*}\right\rangle$. Then, from (3.14) and Lemma 2.4, we have that $x \in F\left(T_{1}\right)$ and $x \in F\left(T_{2}\right)$. Therefore, by Lemma 2.2 , we immediately obtain that

$$
\begin{aligned}
\limsup _{n \rightarrow \infty}\left\langle u-x^{*}, x_{n+1}-x^{*}\right\rangle & =\lim _{i \rightarrow \infty}\left\langle u-x^{*}, x_{n_{i}+1}-x^{*}\right\rangle \\
& =\left\langle u-x^{*}, x-x^{*}\right\rangle \leq 0 .
\end{aligned}
$$

Then it follows from (3.13), (3.15) and Lemma 2.3 that $\left\|x_{n}-x^{*}\right\| \rightarrow 0$ as $n \rightarrow \infty$. Consequently, $x_{n} \rightarrow x^{*}=P_{\mathcal{F}}(u)$.

Case 2. Suppose that there exists a subsequence $\left\{n_{i}\right\}$ of $\{n\}$ such that

$$
\left\|x_{n_{i}}-x^{*}\right\|<\left\|x_{n_{i}+1}-x^{*}\right\|
$$

for all $i \in \mathbb{N}$. Then, by Lemma 2.5, there exists a nondecreasing sequence $\left\{m_{k}\right\} \subset \mathbb{N}$ such that $m_{k} \rightarrow \infty$, and

$$
\left\|x_{m_{k}}-x^{*}\right\| \leq\left\|x_{m_{k}+1}-x^{*}\right\| \quad \text { and } \quad\left\|x_{k}-x^{*}\right\| \leq\left\|x_{m_{k}+1}-x^{*}\right\|
$$

for all $k \in \mathbb{N}$. Now, from (3.12), (3.8), (3.9) and (3.10), we get that $x_{m_{k}}-T_{1} x_{m_{k}} \rightarrow 0$ and $x_{n_{k}}-T_{2} x_{n_{k}} \rightarrow 0$ as $k \rightarrow \infty$. Thus, like in Case 1 , we obtain that

$$
\limsup _{k \rightarrow \infty}\left\langle u-x^{*}, x_{m_{k}+1}-x^{*}\right\rangle \leq 0
$$

Now, from (3.13) we have that

$$
\left\|x_{m_{k}+1}-x^{*}\right\|^{2} \leq\left(1-\alpha_{m_{k}}\right)\left\|x_{m_{k}}-x^{*}\right\|^{2}+2 \alpha_{m_{k}}\left\langle u-x^{*}, x_{m_{k}+1}-x^{*}\right\rangle,
$$


and hence (3.16) and (3.18) imply that

$$
\begin{aligned}
\alpha_{m_{k}}\left\|x_{m_{k}}-x^{*}\right\|^{2} & \leq\left\|x_{m_{k}}-x^{*}\right\|^{2}-\left\|x_{m_{k}+1}-x^{*}\right\|^{2}+2 \alpha_{m_{k}}\left\langle u-x^{*}, x_{m_{k}+1}-x^{*}\right\rangle \\
& \leq+2 \alpha_{m_{k}}\left\langle u-x^{*}, x_{m_{k}+1}-x^{*}\right\rangle .
\end{aligned}
$$

But using the fact that $\alpha_{m_{k}}>0$ and (3.17), we obtain that

$$
\limsup _{k \rightarrow \infty}\left\|x_{m_{k}}-x^{*}\right\|^{2} \leq 0
$$

and hence $\left\|x_{m_{k}}-x^{*}\right\| \rightarrow 0$ as $k \rightarrow \infty$. This together with (3.18) implies that $\left\|x_{m_{k}+1}-x^{*}\right\| \rightarrow$ 0 as $k \rightarrow \infty$. But $\left\|x_{k}-x^{*}\right\| \leq\left\|x_{m_{k}+1}-x^{*}\right\|$ for all $k \in N$, thus we obtain that $x_{k} \rightarrow x^{*}$. Therefore, from the above two cases, we can conclude that $\left\{x_{n}\right\}$ converges strongly to a common fixed point of $T_{1}$ and $T_{2}$ nearest to $u$. The proof is complete.

We note that the method of the proof of Theorem 3.1 provides a convergence theorem for a finite family of Lipschitzian pseudocontractive mappings. In fact, we have the following theorem.

Theorem 3.2 Let $C$ be a nonempty, closed and convex subset of a real Hilbert space $H$. Let $T_{i}: C \rightarrow C, i=1,2, \ldots, N$, be Lipschitz pseudocontractive mappings with Lipschitz constants $L_{i}, i=1,2, \ldots, N$, respectively. Assume that $\mathcal{F}=\bigcap_{i}^{N} F\left(T_{i}\right)$ is nonempty. Let a sequence $\left\{x_{n}\right\}$ be generated from an arbitrary $x_{0}, u \in C$ by

$$
\left\{\begin{array}{l}
y_{n i}=\left(1-\beta_{n}\right) x_{n}+\beta_{n} T_{i} x_{n}, \quad i=1,2, \ldots, N \\
x_{n+1}=\alpha_{n} u+\left(1-\alpha_{n}\right)\left(\theta_{n 0} x_{n}+\sum_{i=1}^{N} \theta_{n i} T_{i} y_{n i}\right),
\end{array}\right.
$$

where $\left\{\theta_{n i}: i=0,1,2, \ldots, N\right\} \subset(a, b) \subset(0,1),\left\{\alpha_{n}\right\} \subset(0, c) \subset(0,1)$ satisfy the following conditions: (i) $\theta_{n 0}+\theta_{n 1}+\cdots+\theta_{n N}=1$; (ii) $\lim _{n \rightarrow \infty} \alpha_{n}=0, \sum \alpha_{n}=\infty$; (iii) $\sum_{i=1}^{N} \theta_{n i} \leq \beta_{n} \leq \beta<$ $\frac{1}{\sqrt{1+L^{2}}+1}, \forall n \geq 1$, for $L:=\max \left\{L_{i}: i=1,2, \ldots, N\right\}$. Then $\left\{x_{n}\right\}$ converges strongly to a common fixed point of $T_{i}(i=1,2, \ldots, N)$ nearest to $u$.

If in Theorem 3.1 we assume that $T$ is nonexpansive, then we have that $T$ is Lipschitz pseudocontractive with $L=1$, and hence we get the following corollary.

Corollary 3.3 Let C be a nonempty, closed and convex subset of a real Hilbert space H. Let $T_{1}, T_{2}: C \rightarrow C$ be nonexpansive mappings. Assume that $\mathcal{F}=F\left(T_{1}\right) \cap F\left(T_{2}\right)$ is nonempty. Let a sequence $\left\{x_{n}\right\}$ be generated from an arbitrary $x_{0}, u \in C$ by

$$
\left\{\begin{array}{l}
z_{n}=\left(1-c_{n}\right) x_{n}+c_{n} T_{2} x_{n} \\
y_{n}=\left(1-\beta_{n}\right) x_{n}+\beta_{n} T_{1} x_{n} \\
x_{n+1}=\alpha_{n} u+\left(1-\alpha_{n}\right)\left(\theta_{n} x_{n}+\delta_{n} T_{1} y_{n}+\gamma_{n} T_{2} z_{n}\right)
\end{array}\right.
$$

where $\left\{\delta_{n}\right\},\left\{\theta_{n}\right\},\left\{\gamma_{n}\right\} \subset(a, b) \subset(0,1),\left\{\alpha_{n}\right\} \subset(0, c) \subset(0,1)$ satisfy the following conditions: (i) $\delta_{n}+\theta_{n}+\gamma_{n}=1$; (ii) $\lim _{n \rightarrow \infty} \alpha_{n}=0, \sum \alpha_{n}=\infty$; (iii) $\delta_{n}+\gamma_{n} \leq c_{n}, \beta_{n} \leq \beta<(\sqrt{2}-1), \forall n \geq 1$. Then $\left\{x_{n}\right\}$ converges strongly to a common fixed point of $T_{1}$ and $T_{2}$ nearest to $u$. 
We now state and prove the convergence theorem for a common zero of a family of monotone mappings.

Corollary 3.4 Let $H$ be a real Hilbert space. Let $A_{i}: H \rightarrow H, i=1,2$, be Lipschitz monotone mappings with Lipschitz constants $L_{1}$ and $L_{2}$, respectively. Assume that $F:=\bigcap_{i=1}^{2} N\left(A_{i}\right)$ is nonempty. Let a sequence $\left\{x_{n}\right\}$ be generated from an arbitrary $x_{1}, u \in H$ by

$$
\left\{\begin{array}{l}
z_{n}=x_{n}-c_{n} A_{2} x_{n} ; \\
y_{n}=x_{n}-\beta_{n} A_{1} x_{n} ; \\
x_{n+1}=\alpha_{n} u+\left(1-\alpha_{n}\right)\left(\theta_{n} x_{n}+\delta_{n}\left(I-A_{1}\right) y_{n}+\gamma_{n}\left(I-A_{2}\right) z_{n}\right),
\end{array}\right.
$$

where $\left\{\delta_{n}\right\},\left\{\theta_{n}\right\},\left\{\gamma_{n}\right\} \subset(a, b) \subset(0,1),\left\{\alpha_{n}\right\} \subset(0, c) \subset(0,1)$ satisfy the following conditions: (i) $\delta_{n}+\theta_{n}+\gamma_{n}=1$; (ii) $\lim _{n \rightarrow \infty} \alpha_{n}=0, \sum \alpha_{n}=\infty$; (iii) $\delta_{n}+\gamma_{n} \leq c_{n}, \beta_{n} \leq \beta<\frac{1}{\sqrt{1+(1+L)^{2}}+1}$, $\forall n \geq 1$, for $L:=\max \left\{L_{1}, L_{2}\right\}$. Then $\left\{x_{n}\right\}$ converges strongly to a common zero point of $A_{1}$ and $A_{2}$ nearest to $u$.

Proof Let $T_{i} x:=\left(I-A_{i}\right) x$ for $i=1,2$. Then we get that every $T_{i}$ for all $i \in\{1,2\}$ is a Lipschitz pseudocontractive mapping with the Lipschitz constant $L_{i}^{\prime}:=\left(1+L_{i}\right)$ and $\bigcap_{i=1}^{2} F\left(T_{i}\right)=$ $\bigcap_{i=1}^{2}\left(A_{i}\right) \neq \emptyset$. Moreover, when $A_{i}$ is replaced with $\left(I-T_{i}\right)$, for each $i \in\{1,2\}$, then scheme (3.21) reduces to scheme (3.1), and hence the conclusion follows from Theorem 3.1.

We may also have the following corollary for a finite family of monotone mappings.

Corollary 3.5 Let $H$ be a real Hilbert space. Let $A_{i}: H \rightarrow H, i=1,2, \ldots, N$, be Lipschitz monotone mappings with Lipschitz constants $L_{i}, i=1,2, \ldots, N$, respectively. Assume that $F:=\bigcap_{i=1}^{N} N\left(A_{i}\right)$ is nonempty. Let a sequence $\left\{x_{n}\right\}$ be generated from an arbitrary $x_{1}, u \in H$ by

$$
\left\{\begin{array}{l}
y_{n i}=x_{n}-\beta_{n} A_{i} x_{n}, \quad i=1,2, \ldots, N \\
x_{n+1}=\alpha_{n} u+\left(1-\alpha_{n}\right)\left(\theta_{n 0} x_{n}+\sum_{i=1}^{N} \theta_{n i}\left(I-A_{i}\right) y_{n i}\right),
\end{array}\right.
$$

where $\left\{\theta_{n i}: i=0,1,2, \ldots, N\right\} \subset(a, b) \subset(0,1),\left\{\alpha_{n}\right\} \subset(0, c) \subset(0,1)$ satisfy the following conditions: (i) $\theta_{n 0}+\theta_{n 1}+\cdots+\theta_{n N}=1$; (ii) $\lim _{n \rightarrow \infty} \alpha_{n}=0, \sum \alpha_{n}=\infty$; (iii) $\sum_{i=1}^{N} \theta_{n i} \leq \beta_{n} \leq \beta<$ $\frac{1}{\sqrt{1+(1+L)^{2}}+1}, \forall n \geq 1$, for $L:=\max \left\{L_{i}: i=1,2, \ldots, N\right\}$. Then $\left\{x_{n}\right\}$ converges strongly to a common zero point of $A_{i}, i=1,2, \ldots, N$, nearest to $u$.

If in Corollary 3.5 we consider a single Lipschitz monotone mapping, then we obtain the following corollary.

Corollary 3.6 Let $H$ be a real Hilbert space. Let $A: H \rightarrow H$ be a Lipschitz monotone mapping with Lipschitz constant L. Assume that $F:=N(A)$ is nonempty. Let a sequence $\left\{x_{n}\right\}$ be generated from an arbitrary $x_{1}, u \in H$ by

$$
\left\{\begin{array}{l}
y_{n}=x_{n}-\beta_{n} A x_{n} \\
x_{n+1}=\alpha_{n} u+\left(1-\alpha_{n}\right)\left(\left(1-\gamma_{n}\right) x_{n}+\gamma_{n}(I-A) y_{n}\right),
\end{array}\right.
$$


where $\left\{\gamma_{n}\right\} \subset(a, b) \subset(0,1),\left\{\alpha_{n}\right\} \subset(0, c) \subset(0,1)$ satisfy the following conditions: (i) $\lim _{n \rightarrow \infty} \alpha_{n}=0, \sum \alpha_{n}=\infty$; (ii) $\gamma_{n} \leq \beta_{n} \leq \beta<\frac{1}{\sqrt{1+(1+L)^{2}}+1}, \forall n \geq 1$. Then $\left\{x_{n}\right\}$ converges strongly to the zero point of $A$ nearest to $u$.

\section{Numerical example}

Now, we give an example of a finite family of pseudocontractive mappings satisfying Theorem 3.1 and some numerical experiment result to explain the conclusion of the theorem as follows.

Example 4.1 Let $H=\mathbb{R}$ with absolute value norm. Let $K=[-2,1]$ and $T_{1}, T_{2}: K \rightarrow K$ be defined by

$$
T_{1} x:= \begin{cases}x+x^{2}, & x \in[-2,0], \\ x, & x \in(0,1]\end{cases}
$$

and

$$
T_{2} x:= \begin{cases}x, & x \in\left[-2, \frac{1}{2}\right], \\ x-2\left(x-\frac{1}{2}\right)^{2}, & x \in\left(\frac{1}{2}, 1\right] .\end{cases}
$$

Clearly, $\mathcal{F}=F\left(T_{1}\right) \cap F\left(T_{2}\right)=[0,1] \cap\left[-2, \frac{1}{2}\right]=\left[0, \frac{1}{2}\right]$, and for $x, y \in K$ we have that

$$
\left\langle\left(I-T_{1}\right) x-\left(I-T_{1}\right) y, x-y\right\rangle \geq 0
$$

and

$$
\left\langle\left(I-T_{2}\right) x-\left(I-T_{2}\right) y, x-y\right\rangle \geq 0,
$$

which show that both mappings are pseudocontractive. Next, we show that $T_{1}$ is Lipschitzian with $L=5$. If $x, y \in[-2,0]$, then

$$
\begin{aligned}
\left|T_{1} x-T_{1} y\right| & =\left|x+x^{2}-y-y^{2}\right| \\
& =|(x+y)+1||x-y| \leq 3|x+y| .
\end{aligned}
$$

If $x, y \in(0,1]$, then

$$
\left|T_{1} x-T_{1} y\right|=|x-y|
$$

If $x \in[-2,0]$ and $y \in(0,1]$, then

$$
\begin{aligned}
\left|T_{1} x-T_{1} y\right| & =\left|x+x^{2}-y\right| \\
& =\left|x-y+x^{2}\right|=\left|x-y+x^{2}-y^{2}+y^{2}\right| \\
& =\left|x-y+x^{2}-y^{2}\right|+y^{2} \\
& \leq|x+y+1| \cdot|x-y|+|y-x| \\
& =|2+x+y| \cdot|x-y| \leq 3|x-y| .
\end{aligned}
$$


If $x \in(0,1]$ and $y \in[-2,0]$, then

$$
\begin{aligned}
\left|T_{1} x-T_{1} y\right| & =\left|x-\left(y+y^{2}\right)\right| \\
& =\left|x-y-y^{2}+x^{2}-x^{2}\right|=\left|x-y+(x+y)(x-y)-x^{2}\right| \\
& =|(1+x+y)||x-y|+x^{2} \\
& \leq|(1+x+y)||x-y|+(x-y)^{2} \\
& \leq(|1+x+y|+|x-y|)|x-y| \\
& \leq 5|x-y| .
\end{aligned}
$$

Thus, we get that $T_{1}$ is Lipschitzian pseudocontractive with $L=5$. Similarly, we can show that $T_{2}$ is Lipschitzian pseudocontractive with $L=10$.

Now, taking $\alpha_{n}=\frac{1}{n+10}, c_{n}=\beta_{n}=\frac{1}{100}+\frac{1}{n+100}, \gamma_{n}=\delta_{n}=0.5\left[\frac{1}{100}+\frac{1}{n+100}\right]$ and $\theta_{n}=1-\left[\frac{1}{100}+\right.$ $\left.\frac{1}{n+100}\right]$, we observe that the conditions of Theorem 3.1 are satisfied and scheme (3.1) provides the data in Tables 1 and 2 and Figures 1 and 2.

(i) When $u=0.6$ and $x_{0}=-1$, we see that the sequence converges to $x^{*}=0.5$ as shown in Table 1 and Figure 1.

(ii) When $u=-1$ and $x_{0}=0.8$, we see that the sequence converges to $x^{*}=0$ as shown in Table 2 and Figure 2.

Table 1 Values of $\left\{x_{n}\right\}$ with initial values $u=0.6$ and $x_{0}=-1$

\begin{tabular}{lllllllll}
\hline $\boldsymbol{n}$ & $\mathbf{0}$ & $\mathbf{5 0 0}$ & $\mathbf{1 , 0 0 0}$ & $\mathbf{5 , 0 0 0}$ & $\mathbf{1 0 , 0 0 0}$ & $\mathbf{1 2 , 0 0 0}$ & $\mathbf{1 4 , 0 0 0}$ & $\mathbf{1 7 , 0 0 0}$ \\
\hline$x_{n}$ & -1.0000 & 0.5639 & 0.5638 & 0.5387 & 0.5287 & 0.5264 & 0.5246 & 0.5225 \\
\hline
\end{tabular}

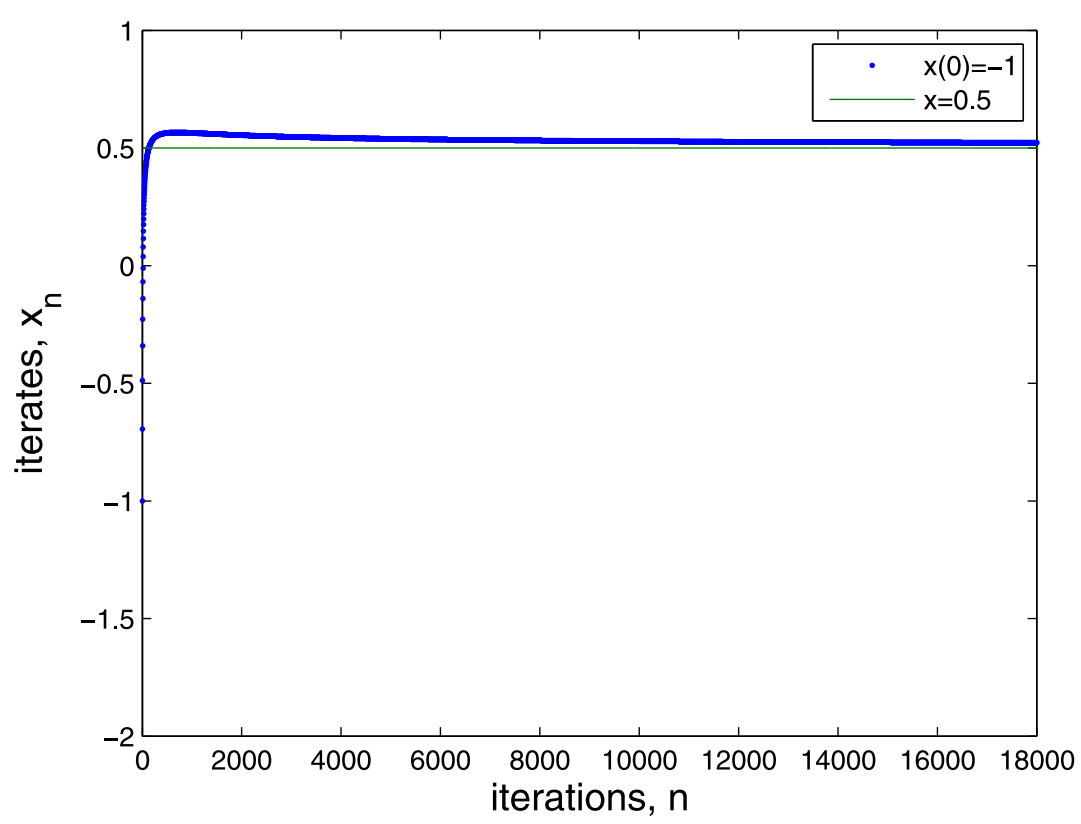

Figure 1 Figure of $\left\{x_{n}\right\}$ with initial values $u=0.6$ and $x_{0}=-1$ 
Table 2 Values of $\left\{x_{n}\right\}$ with initial values $u=-1$ and $x_{0}=0.8$

\begin{tabular}{lllllllll}
\hline $\boldsymbol{n}$ & $\mathbf{0}$ & $\mathbf{5 0 0}$ & $\mathbf{1 , 0 0 0}$ & $\mathbf{5 , 0 0 0}$ & $\mathbf{1 0 , 0 0 0}$ & $\mathbf{1 2 , 0 0 0}$ & $\mathbf{1 4 , 0 0 0}$ & $\mathbf{1 7 , 0 0 0}$ \\
\hline$x_{n}$ & 0.8000 & -0.4781 & -0.3738 & -0.1870 & -0.1351 & -0.1238 & -0.1150 & -0.1047 \\
\hline
\end{tabular}

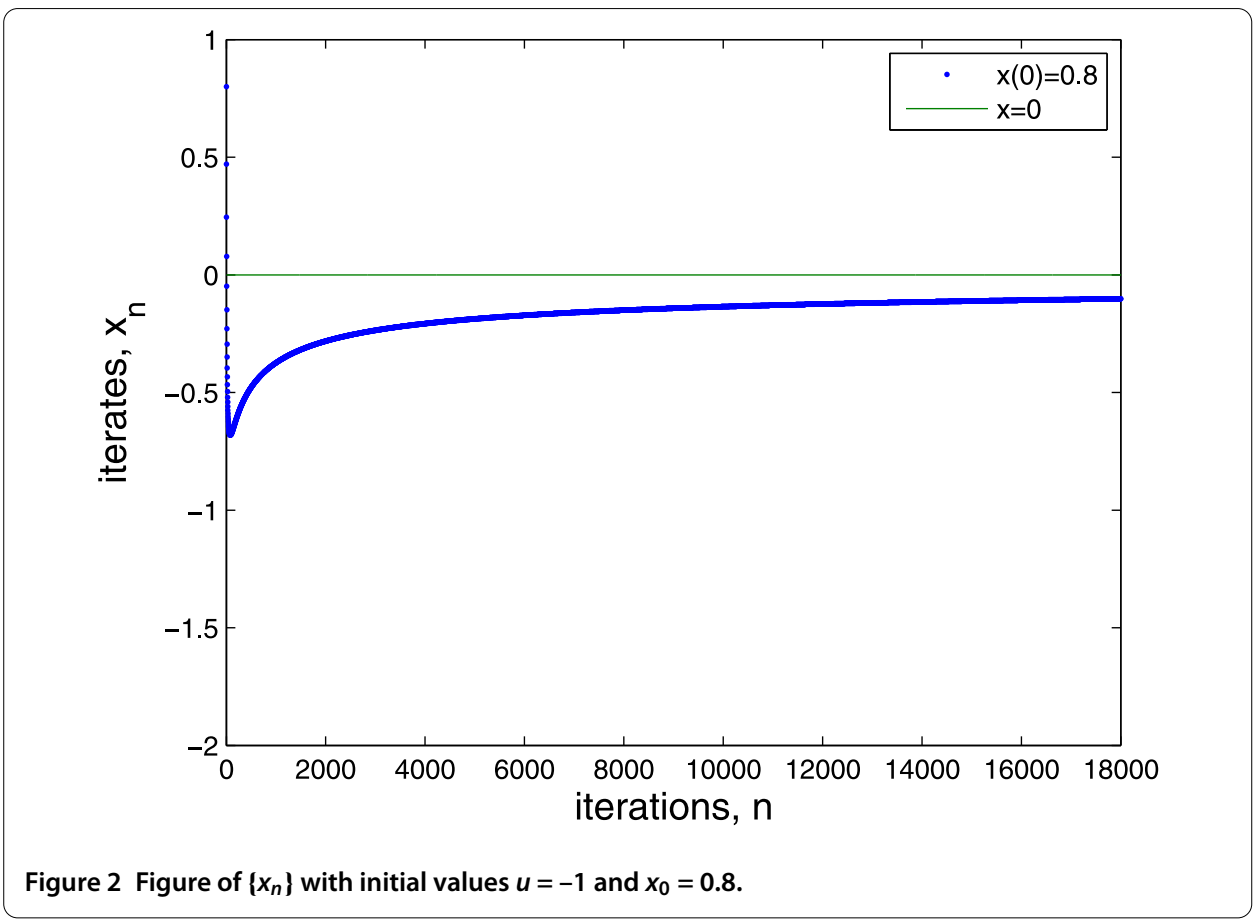

Remark 4.2 Theorem 3.1 provides a convergence sequence to a common fixed point of two Lipschitzian pseudocontractive mappings, whereas Theorem 3.2 provides a convergence sequence to a common fixed point of a finite family of Lipschitzian pseudocontractive mappings. In addition, Corollary 3.4 provides a convergence sequence to a common zero of two Lipschitzian monotone mappings, whereas Theorem 3.5 provides a convergence sequence to a common zero of a finite family of Lipschitzian monotone mappings.

Remark 4.3 Theorem 3.2 improves Theorem ZSA, Theorem 3.2 of Daman and Zegeye [19] in the sense that our convergence does not require the assumption that interior of $F(T)$ is nonempty or condition $(H)$.

Remark 4.4 Theorem 3.1 improves Theorem 3.1 of Zhou [20], Theorem 3.1 of Yao et al. [26] and Theorem 3.1 of Tang et al. [27] in the sense that our convergence requires neither compactness of $T$ nor computation of closed and convex $C_{n}$ of $C$ for each $n \geq 1$.

Competing interests

The authors declare that they have no competing interests.

Authors' contributions

All the authors contributed equally. All authors read and approved the final manuscript.

Author details

${ }^{1}$ Department of Mathematics, University of Botswana, Pvt. Bag 00704, Gaborone, Botswana. ${ }^{2}$ Department of Mathematics, King Abdulaziz University, P.O. Box 80203, Jeddah, 21589, Saudi Arabia. 


\section{Acknowledgements}

This article was funded by the Deanship of Scientific Research (DSR), King Abdulaziz University, Jeddah. The second author acknowledges with thanks DSR for financial support.

Received: 8 April 2013 Accepted: 15 August 2013 Published: 5 September 2013

\section{References}

1. Browder, FE, Petryshyn, WV: Construction of fixed points of nonlinear mappings in Hilbert space. J. Math. Anal. Appl. 20, 197-228 (1967)

2. Zeidler, E: Nonlinear Functional Analysis and Its Applications, Part II: Monotone Operators. Springer, Berlin (1985)

3. Bauschke, HH, Borwein, JM: On projection algorithms for solving convex feasibility problems. SIAM Rev. 38, 367-426 (1996)

4. Combettes, PL: The foundations of set theoretic estimation. Proc. IEEE 81, 182-208 (1993)

5. Bauschke, HH: The approximation of fixed points of compositions of nonexpansive mappings in Hilbert spaces. J. Math. Anal. Appl. 202, 150-159 (1996)

6. Halpern, B: Fixed points of nonexpansive maps. Bull. Am. Math. Soc. 73, 957-961 (1967)

7. Colao, V, Marino, G, Xu, HK: An iterative method for finding common solutions of equilibrium and fixed point problems. J. Math. Anal. Appl. 344, 340-352 (2008)

8. Yao, Y: A general iterative method for a finite family of nonexpansive mappings. Nonlinear Anal. 66, 2676-2687 (2007)

9. Takahashi, S, Takahashi, W: Viscosity approximation methods for equilibrium problems and fixed point problems in Hilbert spaces. J. Math. Anal. Appl. 331, 506-515 (2007)

10. Plubtieng, S, Punpaeng, R: A general iterative method for equilibrium problems and fixed point problems in Hilbert spaces. J. Math. Anal. Appl. 336, 455-469 (2007)

11. Ceng, LC, Cubiotti, P, Yao, JC: Strong convergence theorems for finitely many nonexpansive mappings and applications. Nonlinear Anal. 67, 1464-1473 (2007)

12. Osilike, MO, Shehu, Y: Cyclic algorithm for common fixed points of finite family of strictly pseudocontractive mappings of Browder-Petryshyn type. Nonlinear Anal. 70, 3575-3583 (2009)

13. Zegeye, $\mathrm{H}$, Shahzad, $\mathrm{N}$ : Strong convergence theorems for a common zero of a countably infinite family of $\lambda$-inverse strongly accretive mappings. Nonlinear Anal. 71, 531-538 (2009)

14. Zegeye, $\mathrm{H}$, Shahzad, $\mathrm{N}$ : Strong convergence theorems for a common zero of a finite family of $m$-accretive mappings. Nonlinear Anal. 66, 1161-1169 (2007)

15. Zhang, Y, Guo, Y: Weak convergence theorems of three iterative methods for strictly pseudocontractive mappings of Browder-Petryshyn type. Fixed Point Theory Appl. 2008, Article ID 672301 (2008)

16. Zhou, $\mathrm{H}$ : Convergence theorems of fixed points for a finite family of Lipschitz pseudo-contractions in Banach spaces. Nonlinear Anal. 68, 2977-2983 (2008)

17. Zegeye, H, Shahzadb, N, Alghamdi, MA: Convergence of Ishikawa's iteration method for pseudocontractive mappings. Nonlinear Anal. 74, 7304-7311 (2011)

18. Ishikawa, S: Fixed points by a new iteration method. Proc. Am. Math. Soc. 44, 147-150 (1974)

19. Daman, $\mathrm{OA}$, Zegeye, $\mathrm{H}$ : Strong convergence theorems for a common fixed point of a finite family of pseudocontractive mappings. Int. J. Math. Math. Sci. 2012, Article ID 405315 (2012). doi:10.1155/2012/405315

20. Zhou, H: Convergence theorems of fixed points for Lipschitz pseudo-contractions in Hilbert spaces. J. Math. Anal. Appl. 343, 546-556 (2008)

21. Alber, Y: Metric and generalized projection operators in Banach spaces: properties and applications. In: Kartsatos, AG (ed.) Theory and Applications of Nonlinear Operators of Accretive and Monotone Type. Lecture Notes in Pure and Appl. Math., vol. 178, pp. 15-50. Dekker, New York (1996)

22. Xu, HK: Another control condition in an iterative method for nonexpansive mappings. Bull. Aust. Math. Soc. 65 109-113(2002)

23. Zhang, QB, Cheng, CZ: Strong convergence theorem for a family of Lipschitz pseudocontractive mappings in a Hilbert space. Math. Comput. Model. 48, 480-485 (2008)

24. Maingé, PE: Strong convergence of projected subgradient methods for nonsmooth and non-strictly convex minimization. Set-Valued Anal. 16, 899-912 (2008)

25. Zegeye, H, Shahzad, N: Convergence of Mann's type iteration method for generalized asymptotically nonexpansive mappings. Comput. Math. Appl. 62, 4007-4014 (2011)

26. Yao, YH, Liou, YC, Marino, G: A hybrid algorithm for pseudo-contractive mappings. Nonlinear Anal. 71, 4997-5002 (2009)

27. Tang, Y-C, Peng, J-G, Liu, L-W: Strong convergence theorem for pseudocontractive mappings in Hilbert spaces. Nonlinear Anal. 74, 380-385 (2011). doi:10.1016/j.na.2010.08.048

doi:10.1186/1687-1812-2013-234

Cite this article as: Zegeye and Shahzad: An algorithm for a common fixed point of a family of pseudocontractive mappings. Fixed Point Theory and Applications 2013 2013:234. 